\title{
Methotrexate polyglutamates as a potential marker of adherence to long-term therapy in children with juvenile idiopathic arthritis and juvenile dermatomyositis: an observational, cross-sectional study
}

\author{
Ahmed F. Hawwa ${ }^{1,2}$, AbdelQader AlBawab ${ }^{1,3}$, Madeleine Rooney ${ }^{4}$, Lucy R. Wedderburn ${ }^{5,6,7}$, \\ Michael W. Beresford ${ }^{8}$ and James C. McElnay ${ }^{1 *}$
}

\begin{abstract}
Introduction: Methotrexate (MTX) is a cornerstone of treatment in a wide variety of inflammatory conditions, including juvenile idiopathic arthritis (JIA) and juvenile dermatomyositis (JDM). However, owing to its narrow therapeutic index and the considerable interpatient variability in clinical response, monitoring of adherence to MTX is important. The present study demonstrates the feasibility of using methotrexate polyglutamates (MTXPGs) as a biomarker to measure adherence to MTX treatment in children with JIA and JDM.

Methods: Data were collected prospectively from a cohort of 48 children (median age 11.5 years) who received oral or subcutaneous (SC) MTX therapy for JIA or JDM. Dried blood spot samples were obtained from children by finger pick at the clinic or via self- or parent-led sampling at home, and they were analysed to determine the variability in MTXPG concentrations and assess adherence to MTX therapy.

Results: Wide fluctuations in MTXPG total concentrations (>2.0-fold variations) were found in 17 patients receiving stable weekly doses of MTX, which is indicative of nonadherence or partial adherence to MTX therapy. Age $(P=0.026)$ and route of administration $(P=0.005)$ were the most important predictors of nonadherence to MTX treatment. In addition, the study showed that MTX dose and route of administration were significantly associated with variations in the distribution of MTXPG subtypes. Higher doses and SC administration of MTX produced higher levels of total MTXPGs and selective accumulation of longer-chain MTXPGs $(P<0.001$ and $P<0.0001$, respectively).
\end{abstract}

Conclusions: Nonadherence to MTX therapy is a significant problem in children with JIA and JDM. The present study suggests that patients with inadequate adherence and/or intolerance to oral MTX may benefit from SC administration of the drug. The clinical utility of MTXPG levels to monitor and optimise adherence to MTX in children has been demonstrated.

Trial registration: ISRCTN Registry identifier: ISRCTN93945409. Registered 2 December 2011.

\footnotetext{
*Correspondence: j.mcelnay@qub.ac.uk

${ }^{1}$ Clinical and Practice Research Group, School of Pharmacy, Queen's

University Belfast, 97 Lisburn Road, BT9 7BL Belfast, UK

Full list of author information is available at the end of the article
}

(c) 2015 Hawwa et al. Open Access This article is distributed under the terms of the Creative Commons Attribution 4.0 International License (http://creativecommons.org/licenses/by/4.0/), which permits unrestricted use, distribution, and reproduction in any medium, provided you give appropriate credit to the original author(s) and the source, provide a link to the Creative Commons license, and indicate if changes were made. The Creative Commons Public Domain Dedication waiver (http://creativecommons.org/publicdomain/zero/1.0/) applies to the data made available in this article, unless otherwise stated. 


\section{Introduction}

Methotrexate (MTX) is an effective disease-modifying antirheumatic drug (DMARD) widely used in children to treat a variety of autoimmune and inflammatory conditions, including juvenile idiopathic arthritis (JIA) and juvenile dermatomyositis (JDM) [1-6]. Owing to its efficacy and cost-effectiveness, MTX is likely to remain a cornerstone of treatment $[5,7]$. However, because of the considerable interpatient variability in clinical response, absorption difficulties and a wide spectrum of side effects, monitoring of adherence to MTX is important [4, 8-11].

MTX is a prodrug activated intracellularly to form methotrexate polyglutamates (MTXPGs) through sequential addition of glutamic acid residues by the enzyme folylpolyglutamate synthase (FPGS) [12, 13]. Within the cell, MTXPGs bind to and inhibit dihydrofolate reductase and other folate pathway enzymes required for purine and pyrimidine synthesis, thereby providing anti-inflammatory effects $[14,15]$. Polyglutamation of MTX promotes its retention intracellularly, resulting in enhanced inhibitory effects against its target enzymes. Compared with MTX itself, long-chain MTXPGs have been shown to confer much more potent inhibition of target enzymes within folate metabolism [13, 16, 17]. The clinical value of monitoring serum concentrations of the parent drug is therefore very limited and generally not practical, because approximately $95 \%$ of MTX dose is metabolised within $24 \mathrm{~h}$ of administration [13, 18, 19]. However, monitoring MTXPGs as a surrogate biomarker for drug exposure offers a potential tool to assess adherence to long-term therapy in patients with chronic inflammatory conditions (due to their long half-life of elimination) [20, 21]. Evidence suggests that MTXPGs may be associated with the efficacy and toxicity of MTX treatment in rheumatoid arthritis, and some investigators have advocated routine monitoring of MTXPGs for that reason [22-25].

Apart from one recent study in children with JIA [26], adherence rates to MTX treatment in paediatric patients with rheumatologic diseases, including JIA and JDM, have not been previously reported. Studies investigating MTX adherence in adult rheumatology patients have been based mainly on prescription data [27-29], electronic pill counts [30] or self-reported measures [31]. Reported nonadherence to medications in adult patients with JIA ranges from 8 to $48 \%$, with rates fluctuating depending on the time of evaluation and method used for adherence assessment [32-37]. Risk factors for lack of adherence could include demographic variables such as the patient's being an adolescent or having low socioeconomic status [32, 34, 38], having concerns about side effects of administered drug or lack of belief about the necessity of treatment [31,39].
In the present study, adherence to MTX treatment in a cohort of children with JIA and JDM was evaluated. A method for measuring MTXPG concentrations using the novel dried blood spot (DBS) sampling technique was developed by our group [40] and applied in the present study to evaluate adherence to prescribed MTX therapy in children. A secondary aim of the study was to characterise the pattern of variability in MTXPG concentrations and determine the clinical variables and biochemical parameters that could predict the observed variability in MTX metabolite levels.

\section{Methods}

\section{Study population}

A total of 49 paediatric patients with JIA or JDM were recruited into this study from three paediatric outpatient rheumatology clinics in three different centres: (1) Musgrave Park Hospital, Belfast, Northern Ireland; (2) Alder Hey Children's NHS Foundation Trust, Liverpool, UK; and (3) Great Ormond Street Hospital for Children NHS Foundation Trust, London. Patients between the ages of 4 and 17 years who were receiving stable MTX weekly doses [oral or subcutaneous (SC)] for at least 2 months were identified for inclusion in the study.

Participants were asked to provide one DBS sample for analysis of MTX metabolite content during a regularly scheduled clinic visit. Samples were collected by finger prick from each child by spotting drops of blood (two spots per child) directly onto a Guthrie card. For children 5 years of age or older, parents and/or older children were also asked to take two additional DBS samples at home 4 weeks apart, dry them overnight at room temperature and mail them to our laboratory in prepaid mailer kits for analysis.

Accurate information about MTX dosing and times of sampling were recorded prospectively during the clinic visit using bespoke collection forms. The following data were also collected from patient case notes and clinical chemistry records and recorded in these collection forms: age, weight, height, medical history (date of diagnosis, other medical conditions and current medications), biochemical parameters, clinical outcome and records of side effects experienced (e.g., nausea and vomiting). For samples obtained at home, the mailer kits included appropriate collection forms mailed to the patient and/or the patient's parent in advance of the sampling time, with clear instructions on how to take the sample and fill in the form.

\section{Ethical considerations}

The study was approved by the Office for Research Ethics Committees in Northern Ireland (reference number 10/NIR03/33). Patients were included in the study 
only after their parents or legal guardians had been fully informed and had signed the study consent form. In addition, verbal or written assent was obtained from older children ( $\geq 6$ years) before enrolment into the study.

\section{Measurement of methotrexate polyglutamate concentrations}

DBS concentrations of MTX active metabolites (MTXPGs up to the fifth order of glutamation, $\mathrm{MTXPG}_{5}$ ) were measured by using a selective and sensitive reverse-phase liquid chromatography-tandem mass spectrometry (LC-MS/MS) methodology that we previously developed and validated [40]. Intraday and interday coefficients of variation were less than $15 \%$, and the limits of detection for individual MTXPGs $\left(\mathrm{MTXPG}_{1-5}\right)$ and total concentration of methotrexate polyglutamate $\left(\mathrm{MTXPG}_{\text {total }}\right)$ were determined at $1.6 \mathrm{nmol} / \mathrm{L}$ and $1.5 \mathrm{nmol} / \mathrm{L}$, respectively.

Differences in MTXPG concentrations between patients were evaluated as a function of age, sex, diagnosis and route of administration (SC or oral). To differentiate the effect of these factors from dose- or weight-related changes, MTXPG concentrations were normalised by administered dose of MTX (per kilogram of patient weight or per square metre of body surface area) and expressed as nanomoles per litre per milligram or nanomoles per litre per milligram per square metre.

\section{Assessment of adherence}

The pattern of variability in MTXPG levels as a function of time was evaluated over two consecutive 4-week intervals during which patients were receiving stable MTX doses. Nonadherence was assumed if a patient had wide variations ( $\geq 2.0$-fold) in MTXPG levels on different occasions while prescribed the same dose of MTX. A ratio of 2.0-fold was chosen as the cutoff point because similar values have been suggested for identifying patients nonadherent to long-term therapy in chronic diseases [41, 42].

\section{Statistical analysis}

Statistical analysis was performed using IBM SPSS version 21 software (IBM SPSS, Armonk, NY, USA). The results were expressed as the median and range values or as frequencies. Descriptive statistics were used to characterise the variability in individual $\mathrm{MTXPG}_{1-5}$ and MTXPG $_{\text {total }}$ concentrations between different groups of patients. Univariate associations between MTXPG concentrations and demographic or clinical characteristics were analysed using the Mann-Whitney $U$ test or the Kruskal-Wallis test, as appropriate. Group differences in the proportion of patients with $\geq 2.0$-fold variation in their MTXPG levels were explored using $\chi^{2}$ tests with 1 degree of freedom or Fisher's exact test, as appropriate. Comparison of the median fold ratio of highest to lowest MTXPG concentration was performed using the Mann-Whitney $U$ test. All analyses were twosided, with $P$ values $<0.05$ considered significant.

\section{Results}

\section{Demographic characteristics of the study population}

Of the 49 patients recruited into the study, 32 (65\%) had an underlying diagnosis of JIA and 17 had JDM. The median age (range) of the population was 11.5 years (4-17 years), and $67 \%$ were female. All patients were being treated with weekly MTX (median $0.31 \mathrm{mg} / \mathrm{kg}$, equivalent to $10.2 \mathrm{mg} / \mathrm{m}^{2}$ ) for long durations (median 2.8 years, 5 months -10 years), and $41 \%$ were receiving SC MTX. One patient did not provide a DBS sample at the clinic and therefore was not included in the analysis. Of the patients included in the analysis $(n=48), 40$ (83 \%) provided at least two DBS samples during the study period and $60 \%$ received oral folic acid supplementation (median dose $5 \mathrm{mg} /$ week). The demographic and clinical characteristics of the study participants are shown in Table 1.

\section{Distribution of methotrexate polyglutamate concentrations and effect of administered dose}

In our cohort of 48 patients with clinic DBS samples, the median $\mathrm{MTXPG}_{\text {total }}$ was $91.5 \mathrm{nmol} / \mathrm{L}$ (range $3.5-$ $470.4 \mathrm{nmol} / \mathrm{L}$ ). Analysis of the correlation between MTXPG $_{\text {total }}$ and individual MTXPG subtypes revealed a very high correlation $(R=0.91, P<0.0001)$ between total MTXPGs and MTX triglutamate $\left(\mathrm{MTXPG}_{3}\right)$. Considerable internal correlations of the concentrations within

Table 1 Demographic and clinical characteristics of study patients

\begin{tabular}{|c|c|}
\hline Characteristics & Values $^{\text {a }}(n=49)$ \\
\hline Age (yr) & $11.5(4-17)$ \\
\hline Sex [females, n (\%)] & $33(67.3 \%)$ \\
\hline Weight (kg) & $41(13.4-119.7)$ \\
\hline Height (cm) & $146(95-178)$ \\
\hline MTX weekly dose (mg) & $13.75(7.5-20)$ \\
\hline MTX weekly dose (mg/kg) & $0.31(0.08-0.67)$ \\
\hline MTX weekly dose $\left(\mathrm{mg} / \mathrm{m}^{2}\right)^{\mathrm{b}}$ & $10.2(4.1-15.5)$ \\
\hline Number of medications prescribed & $5(1-8)$ \\
\hline Number of side effects recorded & $3(1-2)$ \\
\hline Pain scale ${ }^{c}$ & $0.9(0-7.1)$ \\
\hline General evaluation scale ${ }^{c}$ & $0.5(0-8.2)$ \\
\hline CMAS score $^{d}$ & $51.5(42-55)$ \\
\hline \multicolumn{2}{|c|}{$\begin{array}{l}\text { aValues represent median (range), except where indicated otherwise } \\
\text { bSurface area was calculated using the Mosteller method [59] } \\
\text { cPain scale and general evaluation scores were obtained from the patients' } \\
\text { Childhood Health Assessment Questionnaire } \\
{ }^{d} \text { Childhood Myositis Assessment Scale (CMAS) score was recorded for children } \\
\text { with JDM }\end{array}$} \\
\hline
\end{tabular}


each MTXPG subgroup were also observed; that is, short-chain MTXPGs MTXPG $_{1-2}$ were highly correlated with each other $(R=0.73, P<0.0001)$, as were very long chain polyglutamates $\mathrm{MTXPG}_{4-5}(R=0.61, P<0.0001)$.

The median concentration of MTXPG total was significantly greater when higher MTX doses were administered $(R=0.27, \quad P=0.0007)$. This appears to be attributable to selective accumulation of long-chain $\mathrm{MTXPG}_{3}(R=0.28, P=0.0005)$ and very long chain MTXPG $_{4-5}(R=0.39-0.42, P<0.0001)$ at the expense of short-chain $\mathrm{MTXPG}_{1-2}$. This was confirmed by the percentage change in individual MTXPGs (relative to MTXPG $\left._{\text {total }}\right)$ as a function of MTX dose (Fig. 1a). Interestingly, concurrent administration of higher folic acid doses was associated with increased concentrations of shorter-chain MTXPG $_{1-2} \quad(R=0.36-0.42, \quad P=0.002)$. Similarly to previous reports [43], the dose of MTX was higher in patients taking folic acid supplementation.

\section{Association of methotrexate polyglutamate levels with clinical variables}

Bivariate analysis revealed significantly higher $\mathrm{MTXPG}_{\text {to- }}$ tal concentrations in patients who had MTX administered by the SC route than among patients who received the drug orally (median $114.1 \mathrm{nmol} / \mathrm{L}$ after SC administration vs. $75.5 \mathrm{nmol} / \mathrm{L}$ after oral administration; $P<$ 0.0001). When corrected for the dose administered (i.e., relative to $1 \mathrm{mg} / \mathrm{kg}$ or $1 \mathrm{mg} / \mathrm{m}^{2}$ weekly doses of MTX), the difference in MTXPG ${ }_{\text {total }}$ concentrations stratified by route of administration remained significant (median concentration $307.8 \mathrm{nmol} / \mathrm{L} / \mathrm{mg} / \mathrm{kg}$ vs. $236.2 \mathrm{nmol} / \mathrm{L} /$ $\mathrm{mg} / \mathrm{kg}$ dose after SC and oral administration, respectively; $P=0.004$; such concentrations are equivalent to $11.0 \mathrm{nmol} / \mathrm{L} / \mathrm{mg} / \mathrm{m}^{2}$ vs. $7.41 \mathrm{nmol} / \mathrm{L} / \mathrm{mg} / \mathrm{m}^{2}$, respectively; $P=0.002$ ).

Furthermore, marked differences in the distribution of individual MTXPG concentrations according to the route of administration were observed. Both normal and dose-adjusted concentrations of long-chain $\mathrm{MTXPG}_{3}$ and longer-chain $\mathrm{MTXPG}_{4-5}$ were higher in patients who received SC MTX than in patients receiving MTX. Evaluation of the proportions of individual MTXPG subtypes (relative to MTXPG $\mathrm{Mtal}_{\text {tol }}$ ) also supported these findings. The median proportions of long-chain $\mathrm{MTXPG}_{3}$ and $\mathrm{MTXPG}_{4}$ were higher in patients treated subcutaneously $\left(P=0.002\right.$ and $P<0.0001$ for $\mathrm{MTXPG}_{3}$ and $\mathrm{MTXPG}_{4}$, respectively), whereas the short-chain $\mathrm{MTXPG}_{1-2}$ made up larger proportions of MTXPGs in patients treated orally $\left(P=0.001\right.$ and $P<0.0001$ for $\mathrm{MTXPG}_{1}$ and $\mathrm{MTXPG}_{2}$, respectively) (Fig. 1b).

MTXPG total concentrations did not change significantly with age $(P>0.05)$, despite the fact that older children received significantly lower doses (per kilogram and per square metre) of MTX $(P<0.0001)$. When corrected for dose administered, however, both MTXPG $_{\text {total }}$ and individual MTXPGs were significantly higher in older children receiving MTX $(P<0.0001$ for MTXPG $_{\text {total }} ; P<0.01$ for MTXPG $_{1-2}$; and $P<0.001$ for $\mathrm{MTXPG}_{3-5}$ ) (Fig. 1c). These findings are indicative of lower clearance rates of MTXPGs in older children than in younger children.

Whether the patient had an underlying diagnosis of JIA or JDM was significantly associated with varying proportions of individual MTXPG subtypes. In particular, patients who had a diagnosis of JIA had significantly greater proportions of short-chain $\mathrm{MTXPG}_{1-2} \quad(P<$ 0.005), whereas long-chain $\mathrm{MTXPG}_{3}$ and very longchain $\mathrm{MTXPG}_{4}$ made up larger proportions of total MTXPGs in patients diagnosed with JDM $(P<0.001$ and $P=0.009$, respectively). Such differences could reflect the fact that higher numbers of patients were treated orally in the JIA subgroup (64\% treated orally in the JIA subgroup vs. $41 \%$ in the JDM subgroup).

\section{Association of methotrexate polyglutamate levels with biochemical parameters}

Increased dose-normalised $\mathrm{MTXPG}_{\text {total }}$ levels were associated with greater antiarthritic effect and improved likelihood of disease control, as suggested by reduced levels of the inflammation markers $C$-reactive protein $(P=0.026)$ and erythrocyte sedimentation rate (ESR; $P=0.033$ ), in patients who had higher accumulation of $\mathrm{MTXPG}_{\text {total }}$ concentrations.

\section{Assessment of adherence to methotrexate therapy}

Highest and lowest MTXPG levels were recorded for each patient who had at least two measurements of their metabolite levels during the study period $(n=40)$. Nonadherence or partial adherence to MTX therapy was identified in 17 (42.5\%) of the patients, in whom greater than 2.0-fold variations in $\mathrm{MTXPG}_{\text {total }}$ concentrations were observed. Of these 17 patients, 5 (29\%) were male and $12(71 \%)$ were female. They ranged in age from 7 to 17 years (median 14 years). At the time the samples were taken, these children were supposed to be taking between 10 and $20 \mathrm{mg}$ weekly MTX doses without change for at least 5 months. Of note, highest to lowest MTXPG ratios, indicative of nonadherence, were significantly greater in patients receiving smaller doses (measured as milligrams per square metre) $(P<0.031)$ (Fig. 2). This suggests that patients with lower disease activity (in whom doses were not increased as they grew) could be associated with an increased risk of poor adherence to therapy. This was particularly apparent when patients were given MTX orally in the present study (Fig. 2). Overall, the results suggest that nearly half of children may fail to fully comply with MTX doses as prescribed during their long-term therapy. 

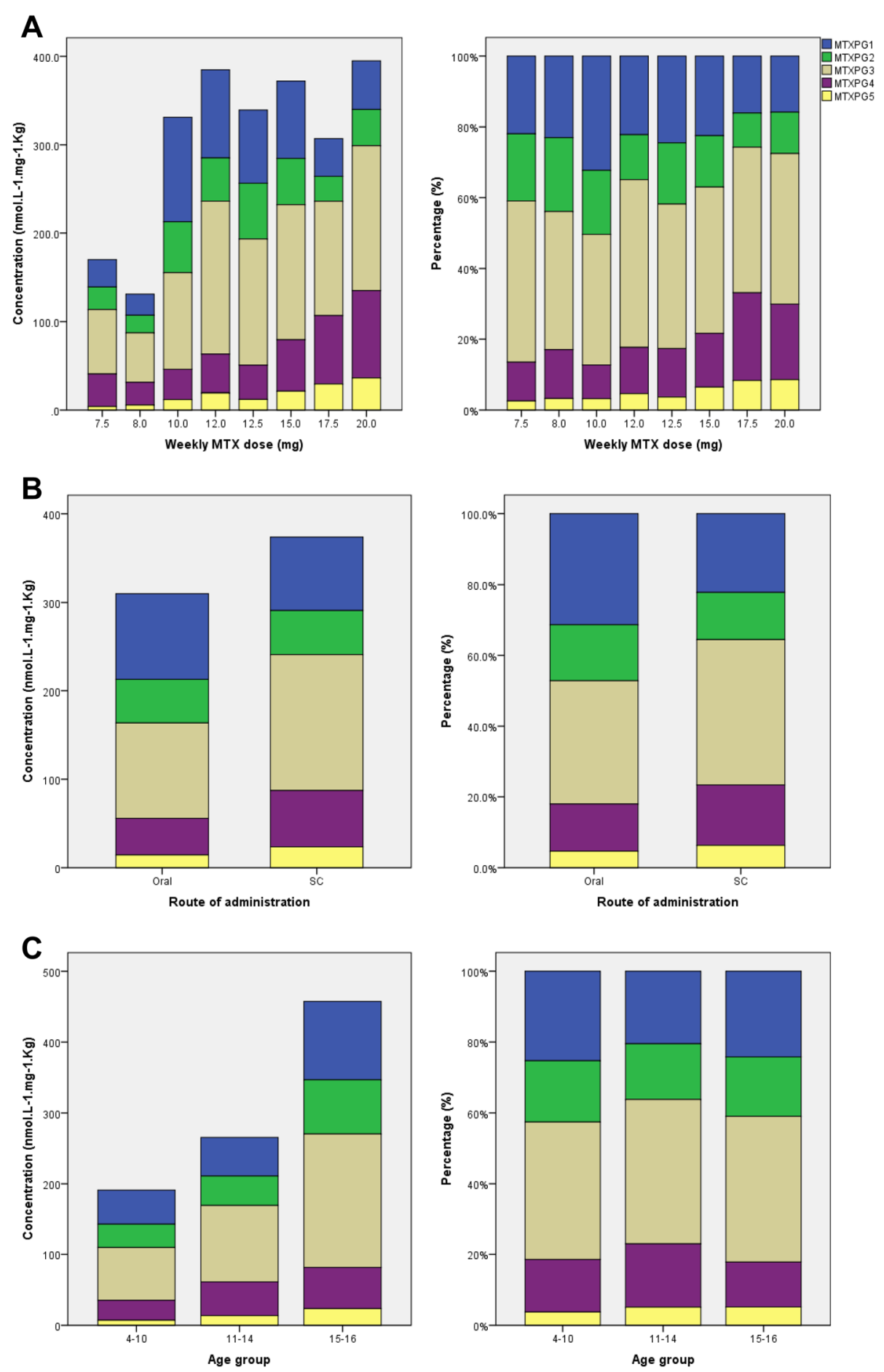

Fig. 1 Effect of administered dose (a), route of administration (b) and patient age (c) on the distribution of methotrexate (MTX) polyglutamates in children receiving long-term MTX therapy

Effect of route of administration and age of child on adherence to methotrexate

Univariate analyses showed that both the route of administration and patient age were significantly associated with nonadherence to MTX treatment (Fig. 2). A higher proportion of patients who received SC MTX were found to be adherent to their medication. Only $17.6 \%$ had greater than 2.0-fold variation in their MTXPG levels after SC administration versus $60.8 \%$ of patients who received MTX orally $(P=0.005)$. 


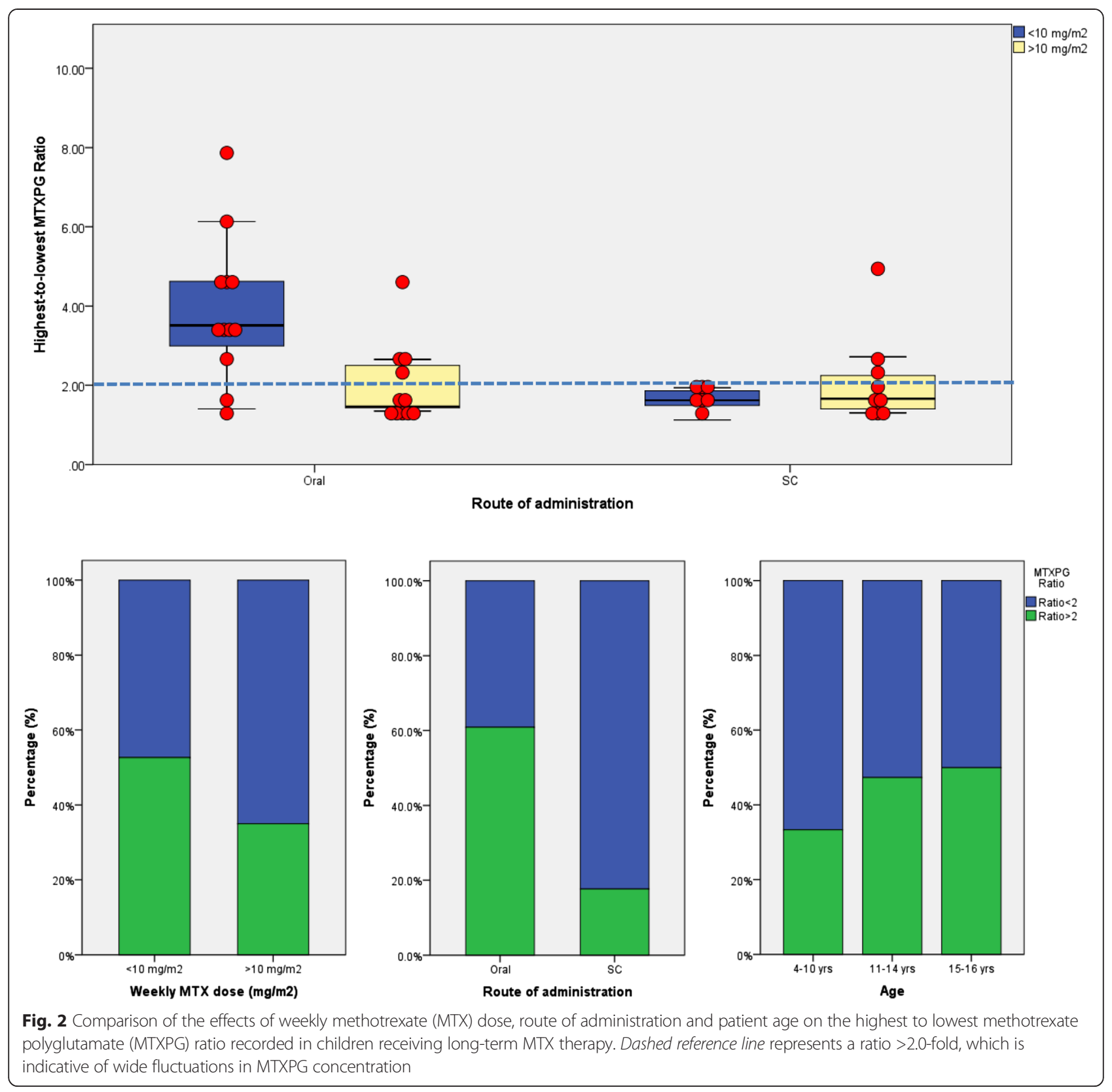

Furthermore, adolescents and older children were more likely than younger children to be nonadherent to their prescribed medication; older age was significantly correlated with greater MTXPG ratio $(R=0.35$, $P=0.026)$.

Sex as a variable was similarly distributed between adherent and nonadherent patients. In addition, there were no statistically significant differences in the underlying diagnosis (JIA vs. JDM) or other obvious sociodemographic characteristics between the nonadherent group and the remainder of the cohort $(P>0.05)$.

\section{Association of nonadherence with biochemical parameters and gastrointestinal toxicity}

The frequency of MTX-related gastrointestinal (GI) side effects (particularly the presence of nausea or vomiting) in nonadherent patients compared with the rest of the cohort were evaluated only in a subset of patients, because reports of nausea and vomiting were recorded only prospectively in 19 patients of those enrolled in the study. Of these 19 patients, 9 reported nausea, vomiting or other GI side effects at some stage during the study. Seven (78\%) of these patients had greater than 2.0-fold 
variation in their MTXPG levels and therefore were considered nonadherent to therapy. The mean MTXPG ratio in patients who reported nausea or vomiting was 3.5-fold compared with 2.4-fold in those who did not have GI complaints. These findings suggest that the presence of MTX-related side effects could increase the risk of poor adherence to MTX in children with JIA and JDM. It is also possible that the nonadherent group did not follow folic acid supplementation prescribed to reduce MTX toxicity.

Finally, examining the differences in biochemical parameters between the adherent and nonadherent groups showed a significant association between nonadherence and elevated ESR levels $(P<0.043)$, suggesting an increased level of inflammation and poor disease control in nonadherent patients. This association was higher in patients receiving MTX orally $(P=0.035)$.

\section{Discussion}

To our knowledge, this is the first study in which the extent of MTX adherence in paediatric patients with JIA and JDM has been evaluated on the basis of DBS measurement of MTXPGs. The approach using DBS proved an acceptable alternative to the use of larger whole blood or red blood cell sample quantities and had the advantage of being minimally invasive, allowing parents and older children to take blood samples at home. The combination of sparse sampling and low sample volume helped to overcome ethical and practical difficulties associated with traditional blood sampling in children and was shown to be useful in estimating adherence.

Similarly to previous reports $[44,45]$, the present study demonstrates significant interpatient variability in total and individual MTXPG concentrations. Such variability was explained, at least in part, by MTX administered dose, route of delivery and patient age. Higher MTX doses were associated with greater percentage of longer-chain $\mathrm{MTXPG}_{3-5}$ at the expense of short-chain MTXPG $_{1-2}$. Such selective enrichment of longer-chain MTXPGs at higher doses is consistent with recent observations in children with JIA and children with acute lymphoblastic leukaemia [45-47]. Similarly, MTXPG distribution differed by the route of administration: $\mathrm{Pa}$ tients receiving SC MTX had higher overall levels of MTXPGs as well as selective accumulation of longerchain MTXPGs. This route-dependent distribution of MTXPGs persisted when concentrations were corrected relative to administered dose (measured as milligrams per kilogram or milligrams per square metre). A plausible explanation for such route-specific and dosedependent accumulation of long-chain MTXPGs could be the higher concentrations produced following SC delivery or higher-dose administration of MTX. It has been suggested that higher concentrations can result in a more pronounced folate-depleted state in cells, which activates a feedback mechanism that upregulates polyglutamation [45]. The stability of longer-chain polyglutamates would in turn allow for the preferential retention and accumulation of long-chain $\mathrm{MTXP}_{3-5}$.

Additional factors likely to be significant contributors to MTXPG variability include pharmacokinetic variation, physiological and developmental differences in oral absorption, transporter expression and the individual genotypes for MTX multiple metabolic pathways that have been associated with both efficacy and toxicity of MTX [48-50]. Because of the importance of folate in growth and development in children, concurrent administration of folic acid supplementation might also explain some of the differences in MTXPG concentrations. This is of particular importance in older children with higher growth demands in puberty.

Folic acid supplementation is routinely given to reduce potential side effects in patients receiving MTX [51, 52]. However, because MTX and folate are transported by the same transporter within cells and compete for FPGS for polyglutamation, it is suggested that higher intracellular folate levels may trump MTX retention and affect its polyglutamation [53, 54]. The present study shows that folic acid use in MTX-treated children was associated with increased concentrations of short-chain MTXPG $_{1-2}$ at the expense of longer-chain MTXPG $_{4-5}$. Such an impact on MTX biotransformation leads to either reduced MTX efficacy or higher MTX dose requirements to achieve the same clinical effect in the absence of folic acid.

Although inherited differences in metabolism may explain interpatient variability, they should not cause intrapatient variability once a steady state is reached. Such variability is hard to explain on any grounds other than not taking the medication as prescribed. Other factors, such as diet and alcohol intake, in teenagers could aggravate this behaviour of nonadherence to prescribed medicines. An approach to detect nonadherence in the present study was therefore to study patients at different times while they were prescribed the same dose of MTX by the same route. If there were a wide variation in MTXPG $\mathrm{total}_{\text {tol }}$ concentrations at steady state, this would most likely be a reflection of poor adherence.

According to this method, nearly half the patients had wide fluctuations in their MTXPG concentrations indicative of partial adherence or nonadherence. Interestingly, doses received by nonadherent children in the present study were significantly lower (measured as milligrams per square metre) than those prescribed to children who were assumed to be adherent to MTX therapy. This increased variability can be explained partly by the children dosed with the lowest oral doses, because such doses will have a greater proportion of 
short-chain MTXPGs that are susceptible to more significant and rapid concentration shifts. It could also indicate that children who received lower doses did not perceive a high necessity for MTX therapy, owing to their low disease activity, and therefore were less likely to take their medication consistently. Conversely, lower doses could indicate an attempt to improve adherence in patients who were more prone to MTX side effects. Further studies are required, however, to confirm these hypotheses.

Other risk factors for nonadherence to MTX therapy include GI side effects associated with the administration of MTX. Seven of the nine patients who reported nausea and vomiting at some stage during the present study had greater than 2.0-fold variation in their MTXPG levels and therefore were considered nonadherent to therapy. Such side effects can be diminished with SC administration of MTX. Patients receiving the drug subcutaneously are therefore expected to show better adherence than those treated orally [55]. Indeed, compared with patients who received the drug orally, a higher proportion of patients who received SC MTX in the present study were found to be adherent to their medication. A possible explanation for better adherence with the SC mode of administration could be the greater antiarthritic effect and better clinical outcome among patients following SC MTX administration compared with those taking oral MTX, as reported in previous studies [55-57]. However, an obvious difference impacting adherence is the fact that SC administration is more likely to be provided by a caregiver and thus is more likely to be observed.

In the present study, adherence did not relate to sex or disease type. However, a significant association between older age of children and nonadherence was established. This is concordant with previous studies that recognised adolescents as having lower adherence than younger children because of their growing autonomy [32, 34, 58]. Older children and adolescents, therefore, should be monitored carefully, particularly when they experience less severe symptoms during the stable phases of their disease. In general, adherent patients had better disease control throughout the study. This was supported by elevated ESR levels, a marker of inflammation, in nonadherent patients.

A precise, sensitive and selective LC-MS/MS assay was used for determination of MTXPGs in the present study. Although LC-MS/MS-based methods are increasingly used in clinical laboratories, the cost involved in sample preparation and access to the instrument might limit its use for routine monitoring of adherence in the clinical setting. Another limitation of the current assay is inability to measure the intracellular levels of relevant folate species. The novel DBS sampling, on the other hand, has the advantage of being both convenient for home sampling and a direct objective measure of adherence. An additional obvious benefit is the lower blood volume requirement applicable for monitoring MTXPGs in children.

\section{Conclusions}

The present study is unique in being the first detailed analysis of MTXPG levels based on DBS measurements in JIA and JDM. Our data highlight the significance of nonadherence to MTX therapy in patients with JIA and JDM and demonstrate the feasibility of measuring MTXPGs in DBS samples as a potential tool to monitor adherence. We also explored the pattern of variability in MTXPG concentrations and its association with clinical variables and additional biochemical parameters pertaining to disease activity and MTX side effects. The findings warrant further investigation into the clinical utility of MTXPG levels to guide therapy and optimise adherence to MTX treatment in children. Furthermore, the study highlights the importance of understanding the potential barriers to MTX adherence in order to help manage the disease and improve patients' quality of life.

\section{Abbreviations}

CMAS: Childhood Myositis Assessment Scale; DBS: Dried blood spot; DMARD: Disease-modifying antirheumatic drug; ESR: Erythrocyte sedimentation rate; FPGS: Folylpolyglutamate synthase; Gl: Gastrointestinal; JDM: Juvenile dermatomyositis; JA: Juvenile idiopathic arthritis; LC-MS/MS: Liquid chromatography-tandem mass spectrometry; MTX: Methotrexate; MTXPG: Methotrexate polyglutamate; MTXPG $_{1-5}$ : Individual methotrexate polyglutamates up to the fifth order of polyglutamation; MTXPGtotal: Total concentration of methotrexate polyglutamate; SC: Subcutaneous.

\section{Competing interests}

The authors declare that they have no competing interests.

\section{Authors' contributions}

JCM, AFH, AA, MR, LRW and MWB contributed to the conception and design of the study and the acquisition and/or interpretation of data. $\mathrm{AA}, \mathrm{AFH}$ and JCM performed the experiments and contributed to the acquisition and/or analysis of data and drafting of the manuscript. JCM, AFH, AA, MR, LRW and MWB substantially contributed to revising the manuscript critically and gave approval of the final manuscript.

\section{Acknowledgements}

The authors acknowledge funding received from Arthritis Research UK (http://www.arthritisresearchuk.org/) under grant 19421. LRW is supported by grants from Great Ormond Street Children's Charity (grant V1304), Arthritis Research UK (grant 20164) and the National Institute for Health Research-funded Biomedical Research Centre Great Ormond Street Hospital NHS Foundation Trust. The funders had no role in the study design, data collection and analysis, decision to publish, or preparation of the manuscript.

\section{Author details}

${ }^{1}$ Clinical and Practice Research Group, School of Pharmacy, Queen's University Belfast, 97 Lisburn Road, BT9 7BL Belfast, UK. ${ }^{2}$ Aston Pharmacy School, Aston University, Birmingham, UK. ${ }^{3}$ Faculty of Pharmacy, Al Zaytoonah University, Amman, Jordan. ${ }^{4}$ Centre for Infection and Immunity, School of Medicine and Biomedical Sciences, Queen's University Belfast, Belfast, UK. ${ }^{5}$ Institute of Child Health, University College London, London, UK. ${ }^{6}$ Arthritis Research UK Centre for Adolescent Rheumatology, University College London, University College London Hospital, London, UK. ${ }^{7}$ Department of Rheumatology, Great Ormond Street Hospital NHS Foundation Trust, London, UK. 'Department of Women's and Children's Health, Institute of Translational Medicine, University of Liverpool, The Alder Hey Children's NHS Foundation Trust, Liverpool, UK. 
Received: 19 March 2015 Accepted: 9 October 2015

\section{Published online: 22 October 2015}

\section{References}

1. Murray KJ, Lovell DJ. Advanced therapy for juvenile arthritis. Best Pract Res Clin Rheumatol. 2002;16:361-78.

2. Saag KG, Teng GG, Patkar NM, Anuntiyo J, Finney C, Curtis JR, et al. American College of Rheumatology 2008 recommendations for the use of nonbiologic and biologic disease-modifying antirheumatic drugs in rheumatoid arthritis. Arthritis Rheum. 2008:59:762-84.

3. Ruperto N, Murray KJ, Gerloni V, Wulffraat N, de Oliveira SK, Falcini F, et al. A randomized trial of parenteral methotrexate comparing an intermediate dose with a higher dose in children with juvenile idiopathic arthritis who failed to respond to standard doses of methotrexate. Arthritis Rheum. 2004:50:2191-201.

4. Lambert CM, Sandhu S, Lochhead A, Hurst NP, McRorie E, Dhillon V. Dose escalation of parenteral methotrexate in active rheumatoid arthritis that has been unresponsive to conventional doses of methotrexate: a randomized, controlled trial. Arthritis Rheum. 2004;50:364-71.

5. Ramanan AV, Whitworth P, Baildam EM. Use of methotrexate in juvenile idiopathic arthritis. Arch Dis Child. 2003;88:197-200.

6. Pilkington CA, Wedderburn LR. Paediatric idiopathic inflammatory muscle disease: recognition and management. Drugs. 2005;65:1355-65.

7. Weinblatt ME, Kaplan H, Germain BF, Block S, Solomon SD, Merriman RC, et al. Methotrexate in rheumatoid arthritis: a five-year prospective multicenter study. Arthritis Rheum. 1994;37:1492-8.

8. Cohen S, Cannon GW, Schiff M, Weaver A, Fox R, Olsen N, et al. Two-year, blinded, randomized, controlled trial of treatment of active rheumatoid arthritis with leflunomide compared with methotrexate. Arthritis Rheum. 2001;44:1984-92.

9. Becker ML, Rosé CD, Cron RQ, Sherry DD, Bilker WB, Lautenbach E. Effectiveness and toxicity of methotrexate in juvenile idiopathic arthritis: comparison of 2 initial dosing regimens. J Rheumatol. 2010;37:870-5.

10. Ortiz-Alvarez O, Morishita K, Avery G, Green J, Petty RE, Tucker LB, et al. Guidelines for blood test monitoring of methotrexate toxicity in juvenile idiopathic arthritis. J Rheumatol. 2004;31:2501-6.

11. Dougados M, Combe B, Cantagrel A, Goupille P, Olive P, Schattenkirchner $M$, et al. Combination therapy in early rheumatoid arthritis: a randomised, controlled, double blind 52 week clinical trial of sulphasalazine and methotrexate compared with the single components. Ann Rheum Dis. 1999;58:220-5.

12. Kremer JM. Toward a better understanding of methotrexate. Arthritis Rheum. 2004;50:1370-82.

13. Chabner BA, Allegra CJ, Curt GA, Clendeninn NJ, Baram J, Koizumi S, et al. Polyglutamation of methotrexate: is methotrexate a prodrug? J Clin Invest. 1985;76:907-12.

14. Cutolo M, Sulli A, Pizzorni C, Seriolo B, Straub RH. Anti-inflammatory mechanisms of methotrexate in rheumatoid arthritis. Ann Rheum Dis. 2001;60:729-35.

15. Cronstein BN, Naime D, Ostad E. The antiinflammatory mechanism of methotrexate. Increased adenosine release at inflamed sites diminishes leukocyte accumulation in an in vivo model of inflammation. J Clin Invest. 1993;92:2675-82.

16. Allegra CJ, Chabner BA, Drake JC, Lutz R, Rodbard D, Jolivet J. Enhanced inhibition of thymidylate synthase by methotrexate polyglutamates. J Biol Chem. 1985;260:9720-6.

17. Schroder H, Fogh K. Methotrexate and its polyglutamate derivatives in erythrocytes during and after weekly low-dose oral methotrexate therapy of children with acute lymphoblastic leukemia. Cancer Chemother Pharmacol. 1988:21:145-9.

18. Bannwarth B, Pehourca F, Schaeverbeke T, Dehais J. Clinical pharmacokinetics of low-dose pulse methotrexate in rheumatoid arthritis. Clin Pharmacokinet. 1996;30:194-210.

19. Ravelli A, Di Fuccia G, Molinaro M, Ramenghi B, Zonta L, Regazzi MB, et al. Plasma levels after oral methotrexate in children with juvenile rheumatoid arthritis. J Rheumatol. 1993;20:1573-7.

20. Dervieux T, Orentas Lein D, Marcelletti J, Pischel K, Smith K, Walsh M, et al. HPLC determination of erythrocyte methotrexate polyglutamates after lowdose methotrexate therapy in patients with rheumatoid arthritis. Clin Chem. 2003;49:1632-41.
21. Dalrymple JM, Stamp LK, O'Donnell JL, Chapman PT, Zhang M, Barclay ML. Pharmacokinetics of oral methotrexate in patients with rheumatoid arthritis. Arthritis Rheum. 2008:58:3299-308.

22. Angelis-Stoforidis P, Vajda FJ, Christophidis N. Methotrexate polyglutamate levels in circulating erythrocytes and polymorphs correlate with clinical efficacy in rheumatoid arthritis. Clin Exp Rheumatol. 1999;17:313-20.

23. Cronstein B. How does methotrexate suppress inflammation? Clin Exp Rheumatol. 2010;28:521-3.

24. Dervieux T, Furst D, Lein DO, Capps R, Smith K, Caldwell J, et al. Pharmacogenetic and metabolite measurements are associated with clinical status in patients with rheumatoid arthritis treated with methotrexate: results of a multicentred cross sectional observational study. Ann Rheum Dis. 2005;64:1180-5.

25. Dervieux T, Furst D, Lein DO, Capps R, Smith K, Walsh M, et al. Polyglutamation of methotrexate with common polymorphisms in reduced folate carrier, aminoimidazole carboxamide ribonucleotide transformylase, and thymidylate synthase are associated with methotrexate effects in rheumatoid arthritis. Arthritis Rheum. 2004;50:2766-74.

26. Pelajo CF, Sgarlat CM, Lopez-Benitez JM, Oliveira SK, Rodrigues MC, Sztajnbok FR, et al. Adherence to methotrexate in juvenile idiopathic arthritis. Rheumatol Int. 2012;32:497-500.

27. Harley CR, Frytak JR, Tandon N. Treatment compliance and dosage administration among rheumatoid arthritis patients receiving infliximab, etanercept, or methotrexate. Am J Manag Care. 2003;9:S136-43.

28. Grijalva CG, Chung CP, Arbogast PG, Stein CM, Mitchel Jr EF, Griffin MR. Assessment of adherence to and persistence on disease-modifying antirheumatic drugs (DMARDs) in patients with rheumatoid arthritis. Med Care. 2007:45:S66-76.

29. Harrold LR, Andrade SE. Medication adherence of patients with selected rheumatic conditions: a systematic review of the literature. Semin Arthritis Rheum. 2009;38:396-402.

30. Waimann CA, Marengo MF, de Achaval S, Cox VL, Garcia-Gonzalez A, Reveille JD, et al. Electronic monitoring of oral therapies in ethnically diverse and economically disadvantaged patients with rheumatoid arthritis: consequences of low adherence. Arthritis Rheum. 2013;65:1421-9.

31. de Thurah A, Norgaard M, Harder I, Stengaard-Pedersen K. Compliance with methotrexate treatment in patients with rheumatoid arthritis: influence of patients' beliefs about the medicine: a prospective cohort study. Rheumatol Int. 2010;30:1441-8.

32. April KT, Feldman DE, Platt RW, Duffy CM. Comparison between children with juvenile idiopathic arthritis and their parents concerning perceived treatment adherence. Arthritis Rheum. 2006;55:558-63.

33. Degotardi PJ, Revenson TA, llowite NT. Family-level coping in juvenile rheumatoid arthritis: assessing the utility of a quantitative family interview. Arthritis Care Res. 1999;12:314-24.

34. Feldman DE, de Civita M, Dobkin PL, Malleson P, Meshefedjian G, Duffy CM. Perceived adherence to prescribed treatment in juvenile idiopathic arthritis over a one-year period. Arthritis Rheum. 2007;57:226-33.

35. Litt IF, Cuskey WR. Compliance with salicylate therapy in adolescents with juvenile rheumatoid arthritis. Am J Dis Child. 1981;135:434-6.

36. Rapoff MA, Belmont JM, Lindsley CB, Olson NY. Electronically monitored adherence to medications by newly diagnosed patients with juvenile rheumatoid arthritis. Arthritis Rheum. 2005;53:905-10.

37. Sturge C, Garralda ME, Boissin M, Dore CJ, Woo P. School attendance and juvenile chronic arthritis. Br J Rheumatol. 1997;36:1218-23.

38. Skoglund KA, Söderhall S, Beck O, Peterson C, Wennberg M, Hayder S, et al. Plasma and urine levels of methotrexate and 7-hydroxymethotrexate in children with ALL during maintenance therapy with weekly oral methotrexate. Med Pediatr Oncol. 1994;22:187-93.

39. Donovan JL, Blake DR. Patient non-compliance: deviance or reasoned decision-making? Soc Sci Med. 1992;34:507-13.

40. Hawwa AF, Albawab A, Rooney M, Wedderburn LR, Beresford MW, McElnay JC. A novel dried blood spot-LCMS method for the quantification of methotrexate polyglutamates as a potential marker for methotrexate use in children. PLoS One. 2014;::e89908.

41. Davies HA, Lennard L, Lilleyman JS. Variable mercaptopurine metabolism in children with leukaemia: a problem of non-compliance? BMJ. 1993;306:1239-40.

42. Hawwa AF, Millership JS, Collier PS, McCarthy A, Dempsey S, Cairns C, et al. The development of an objective methodology to measure medication adherence to oral thiopurines in paediatric patients with acute 
lymphoblastic leukaemia_an exploratory study. Eur J Clin Pharmacol. 2009:65:1105-12.

43. van Ede AE, Laan RF, Rood MJ, Huizinga TW, van de Laar MA, van Denderen CJ, et al. Effect of folic or folinic acid supplementation on the toxicity and efficacy of methotrexate in rheumatoid arthritis: a forty-eight week, multicenter, randomized, double-blind, placebo-controlled study. Arthritis Rheum. 2001:44:1515-24

44. Stamp LK, O'Donnell JL, Chapman PT, Zhang M, Frampton C, James J, et al. Determinants of red blood cell methotrexate polyglutamate concentrations in rheumatoid arthritis patients receiving long-term methotrexate treatment. Arthritis Rheum. 2009;60:2248-56.

45. Becker ML, van Haandel L, Gaedigk R, Lasky A, Hoeltzel M, Stobaugh J, et al. Analysis of intracellular methotrexate polyglutamates in patients with juvenile idiopathic arthritis: effect of route of administration on variability in intracellular methotrexate polyglutamate concentrations. Arthritis Rheum. 2010;62:1803-12

46. Adam de Beaumais T, Dervieux T, Fakhoury M, Medard Y, Azougagh S, Zhang $D$, et al. The impact of high-dose methotrexate on intracellular 6-mercaptopurine disposition during interval therapy of childhood acute lymphoblastic leukemia. Cancer Chemother Pharmacol. 2010;66:653-8.

47. Dervieux T, Zablocki R, Kremer J. Red blood cell methotrexate polyglutamates emerge as a function of dosage intensity and route of administration during pulse methotrexate therapy in rheumatoid arthritis. Rheumatology (Oxford). 2010:49:2337-45.

48. Campalani E, Arenas M, Marinaki AM, Lewis CM, Barker JN, Smith CH. Polymorphisms in folate, pyrimidine, and purine metabolism are associated with efficacy and toxicity of methotrexate in psoriasis. J Invest Dermatol. 2007:127:1860-7

49. Warren RB, Smith RL, Campalani E, Eyre S, Smith CH, Barker JN, et al. Genetic variation in efflux transporters influences outcome to methotrexate therapy in patients with psoriasis. J Invest Dermatol. 2008;128:1925-9.

50. Warren RB, Smith RL, Campalani E, Eyre S, Smith CH, Barker JN, et al. Outcomes of methotrexate therapy for psoriasis and relationship to genetic polymorphisms. Br J Dermatol. 2009;160:438-41.

51. Ortiz Z, Shea B, Suarez Almazor M, Moher D, Wells G, Tugwell P. Folic acid and folinic acid for reducing side effects in patients receiving methotrexate for rheumatoid arthritis. Cochrane Database Syst Rev. 2000;2:CD000951.

52. Ortiz Z, Shea B, Suarez-Almazor ME, Moher D, Wells GA, Tugwell P. The efficacy of folic acid and folinic acid in reducing methotrexate gastrointestinal toxicity in rheumatoid arthritis: a metaanalysis of randomized controlled trials. J Rheumatol. 1998;25:36-43.

53. Jolivet J, Faucher F, Pinard MF. Influence of intracellular folates on methotrexate metabolism and cytotoxicity. Biochem Pharmacol. 1987;36:3310-2.

54. Kennedy DG, Van den Berg HW, Clarke R, Murphy RF. The effect of leucovorin on the synthesis of methotrexate poly-y-glutamates in the MCF-7 human breast cancer cell line. Biochem Pharmacol. 1985;34:2897-903.

55. Braun J, Kästner P, Flaxenberg P, Währisch J, Hanke P, Demary W, et al. Comparison of the clinical efficacy and safety of subcutaneous versus oral administration of methotrexate in patients with active rheumatoid arthritis: results of a six-month, multicenter, randomized, double-blind, controlled phase IV trial. Arthritis Rheum. 2008;58:73-81.

56. Hamilton RA, Kremer JM. Why intramuscular methotrexate may be more efficacious than oral dosing in patients with rheumatoid arthritis. $\mathrm{Br} J$ Rheumatol. 1997;36:86-90

57. Todd DJ, Weinblatt ME. Oral versus parenteral administration of methotrexate for the treatment of active RA. Nat Clin Pract Rheumatol. 2008;4:342-3.

58. Staples B, Bravender T. Drug compliance in adolescents: assessing and managing modifiable risk factors. Paediatr Drugs. 2002;4:503-13.

59. Mosteller RD. Simplified calculation of body-surface area. N Engl J Med. 1987:317:1098.

\section{Submit your next manuscript to BioMed Central and take full advantage of:}

- Convenient online submission

- Thorough peer review

- No space constraints or color figure charges

- Immediate publication on acceptance

- Inclusion in PubMed, CAS, Scopus and Google Scholar

- Research which is freely available for redistribution

Submit your manuscript at www.biomedcentral.com/submit 